\title{
ATENÇÃO ONCOLÓGICA NA ATENÇÃO BÁSICA: PROJETO DE EXTENSÃO NA FORMAÇÃO DE ACADÊMICOS DE ENFERMAGEM
}

\author{
Luciana Martins da Rosa \\ Universidade Federal de Santa Catarina \\ luciana.m.rosa@ufsc.br \\ Amanda Espíndola de Andrade \\ Universidade Federal de Santa Catarina \\ amandandrade.esp@gmail.com \\ Luiza Kretzer Berndt \\ Universidade Federal de Santa Catarina \\ luizakretzerb@gmail.com
}

\author{
Jane Cristina Anders \\ Universidade Federal de Santa Catarina \\ jane.anders@ufsc.br \\ Vera Radünz \\ Universidade Federal de Santa Catarina \\ radunz@ccs.ufsc.br \\ Ana Izabel Jatobá de Souza \\ Universidade Federal de Santa Catarina \\ jatoba.izabel@ufsc.br
}

\begin{abstract}
Resumo
Este estudo tem como objetivo identificar como os acadêmicos de enfermagem, bolsistas de um projeto de extensão percebem e avaliam a aprendizagem e a contribuição para o exercício profissional. Trata-se de um estudo descritivo com abordagem qualitativa, incluindo sete acadêmicos de enfermagem, participantes do projeto de extensão que abrangeu as demandas de educação permanente para atenção oncológica na atenção básica de Florianópolis. A coleta de dados ocorreu por meio de aplicação de questionário, em setembro de 2016. Os dados coletados foram submetidos à análise de conteúdo. As categorias estabelecidas foram: Contribuição para formação em enfermagem; Contribuição para a educação permanente em enfermagem e para a atenção oncológica na atenção básica em saúde; Problematização da atuação do enfermeiro na atenção básica em saúde e formação acadêmica. Os bolsistas apontaram a efetividade da participação em projeto de extensão como estratégia formativa.
\end{abstract}

Palavras-chave: Educação Superior. Oncologia. Enfermagem. Extensão Comunitária.

\section{ONCOLOGIC AT'TENTION IN PRIMARY HELTH CARE: EXTENSION PROJECT IN NURSING EDUCATION}

\section{Abstract}

This study aims to identify how nursing students, as fellows of the extensión project perceive and evaluate the learning and the contribution to profesional practice A descriptive study was conducted with a qualitative approach, including seven nursing students, participants of the extensión project that covered the demands of permanent education for cancer care in the basic care of Florianópolis. The data was collected through a questionnaire in September 2016. The data collected was subjected to contentan alysis. The established categories: were: Contribution to nursing education; Contribution to the continuing education of nursing and in primary healthcare; Problematization of nurses performance in basic health care and nursing formation. Fellows showed the effective ness of participation in extensión project as a formative strategy.

Keywords: College Education. Oncology. Nursing. Community Outreach.

\section{ATENCIÓN ONCOLÓGICA EN ATENCIÓN PRIMARIA: PROYECTO DE EXTENSIÓN EN LA FORMACIÓN ACADÉMICA EN ENFERMERÍA}

\section{Resumen}

Este estudio tiene como objetivo identificar cómo los estudiantes de enfermería perciben y evalúan la aprendizaje y la contribución de las actividades de uno proyecto de extensión para la práctica profesional. Estudio descriptivo con un enfoque cualitativo, incluyendo siete estudiantes de enfermería participantes en el proyecto de extensión que abarcó las demandas de educación permanente para atención oncológica en la atención básica de Florianópolis. Los datos fueron recolectados a través de cuestionario, en septiembre de 2016 y sometidos a análisis de contenido. Las categorías establecidas fueron: Contribución a la formación en enfermería; Contribución a la formación continuada de enfermería y cuidado del cáncer en la atención primaria de la salud; Problematización de la actuación de las enfermeras en la atención primaria de la salud y la educación académica. Los estudiantes mostraron la eficacia de la participación en el proyecto de extensión como estrategia formativa.

Palabras chaves: Educación Superior. Oncología. Enfermería. Extensión a la Comunidad. 
Atenção oncológica na atenção básica: projeto de extensão na formação de acadêmicos de enfermagem

\section{INTRODUÇÃO}

O perfil epidemiológico do câncer no Brasil e no Mundo chama a atenção dos governantes e das sociedades, principalmente devido as previsões de incidência e prevalência para as próximas décadas. Estima-se que, para o Brasil, a incidência da doença entre 2016 e 2030 aumentará cerca de 30\%. A incidência atual é de cerca de 600 mil casos novos a cada ano e a prevalência de 1 milhão de casos. (ERVIK et al., 2016; INSTITUTO NACIONAL DO CÂNCER, 2015).

Este panorama vem exigindo que os profissionais atuantes nos diferentes níveis de atenção à saúde estejam preparados para o atendimento de pessoas com suspeita de câncer, com doença oncológica e dos sobreviventes do câncer. No entanto, estudo realizado pelo Instituto Nacional do Câncer (INCA) aponta o déficit de qualificação dos profissionais da área da saúde e, destaca que esta limitação é ainda maior dentre os profissionais atuantes na atenção básica. (THULER; BERGMANN, 2011).

Estudo realizado com enfermeiros (77) atuantes na atenção básica verificou que a maioria dos profissionais desconhece a política de atenção oncológica e maioria manifestou dificuldades para realização do cuidado de enfermagem, sendo a falta de capacitação em oncologia o motivo preponderante para esta dificuldade. (SOUZA; CAZOLA; OLIVEIRA, 2017).

A qualificação profissional envolve o desenvolvimento de habilidades relacionais ao manejo dos pacientes e seus familiares, a partir de um modo propositivo de enfrentamento do tratamento e de atenção à sobrevivência ao câncer, que conduz o profissional a aprender a lidar com os medos, sofrimentos, necessidades psicobiológicas, espirituais e de saúde/doença. (LUZ, 2016).

Preocupados com esta realidade, um grupo de professores e acadêmicos do Curso de Graduação em Enfermagem da Universidade Federal de Santa Catarina (UFSC) foi desenvolvido um projeto de extensão, que incluiu atividades de pesquisa, para contribuir com a redução dos déficits de qualificação dos profissionais de enfermagem da atenção básica do município de Florianópolis-SC (Brasil).

Este projeto foi intitulado: “Atenção oncológica na Atenção Básica de Florianópolis: a Enfermagem da UFSC auxiliando os enfermeiros nas demandas de qualificação", e tem como objetivos principais: identificar as demandas de qualificação em Oncologia para os enfermeiros atuantes na atenção básica no município de Florianópolis; desenvolver Objeto Virtual de Aprendizagem (OVA) para favorecer o aprendizado e o aprimoramento técnico e científico dos 
Atenção oncológica na atenção básica: projeto de extensão na formação de acadêmicos de enfermagem

enfermeiros e equipe de enfermagem, contendo as demandas de qualificação em Oncologia identificadas na investigação com os enfermeiros.

$\mathrm{Na}$ abordagem do paciente com câncer e seus familiares, a comunicação e a informação adequada, são determinantes para a qualidade do cuidado (LUZ, 2015). Assim, quanto maior conhecimento por parte dos profissionais melhores resultados podem ser alcançados no controle do câncer e suas complicações.

O projeto foi realizado nos cinco Distritos Sanitários de Saúde e em 55 Centros de Saúde de Florianópolis desde abril de 2015. Foram incluídos 84 enfermeiros na coleta de dados $(52 \%$ do total de enfermeiros atuantes no cenário). O ambiente virtual foi disponibilizado para todos os profissionais de enfermagem da atenção básica, cerca de 500 profissionais (incluindo enfermeiros, técnicos de enfermagem e auxiliares de enfermagem).

A participação dos acadêmicos ocorreu como bolsistas, sendo que as atividades desempenhadas envolveram organização e desenvolvimento da coleta de dados relacionadas aos déficits de qualificação para atendimento das demandas oncológicas na atenção básica em saúde e, a partir desta identificação a elaboração de material educativo para aprimoramento técnico e científico dos profissionais, disponibilizados em OVA. Os professores atuantes no projeto orientaram e acompanharam o desenvolvimento das atividades dos bolsistas, estimulando o exercício profissional relacionado à atuação como enfermeiros e como pesquisadores no contexto da saúde.

A atuação como bolsistas permitiu o envolvimento intenso com as atividades e as estratégias propostas, redobrando a atenção, a participação, o envolvimento, o desejo de aprender para ensinar, definindo metas e objetivos a serem atingidos. (PRANKE; FRISON, 2015). Assim, adquirindo conhecimento, novas competências, conhecendo as necessidades do exercício da profissão, tanto de atuação como para o desenvolvimento de pesquisa e para o aprimoramento da Enfermagem. Os professores integrantes deste projeto são experts em Oncologia e em ambiente virtual de aprendizagem e, os bolsistas, acadêmicos de enfermagem são acadêmicos inexperientes na temática em questão, bem como no desenvolvimento de pesquisas.

Para este estudo questiona-se: qual a percepção dos acadêmicos de enfermagem sobre a aprendizagem alcançada com o desenvolvimento deste projeto? Quais as atividades realizadas pelos acadêmicos de enfermagem durante o desenvolvimento do estudo? Qual a avaliação do acadêmico de enfermagem sobre a atividade de extensão realizada?

Justifica-se o desenvolvimento do projeto de extensão pelas argumentações já apresentadas e porque uma das funções das instituições de ensino superior é possibilitar a aprendizagem sobre o desenvolvimento de atividades de extensão e de pesquisa, favorecendo o 
Atenção oncológica na atenção básica: projeto de extensão na formação de acadêmicos de enfermagem

desenvolvimento de competências, para que eles possam gerir seus processos de aprendizagem e trilhar, com autonomia crescente, seu percurso acadêmico, dispondo de ferramentas intelectuais e sociais que lhes permitam a aprendizagem ao longo da vida. Assim, este estudo procura avaliar a prática de ensino e pesquisa realizada, pois, os professores precisam analisar os próprios estilos de aprendizagem, avaliar sua compreensão, tomar consciência do que realmente é importante ensinar e de que maneira proceder para que o aluno aprenda conscientemente. (PRANKE; FRISON, 2015).

Portanto, firma-se como objetivo deste estudo: identificar como os acadêmicos de enfermagem, bolsistas de um projeto de extensão percebem e avaliam a aprendizagem e a contribuição para o exercício profissional.

\section{MÉTODOS}

Trata-se de um estudo descritivo com abordagem qualitativa, realizado no Departamento de Enfermagem da UFSC e aprovado pelo Comitê de Ética da UFSC sob o Certificado de Apresentação para Apreciação Ética (CAAE) número 45895615.2.0000.0121.

Foram incluídos sete acadêmicos de enfermagem participantes do projeto de extensão, sendo quatro bolsistas atuantes no primeiro ano do desenvolvimento do projeto (abril de 2015 março de 2017); uma do segundo ano (abril de 2016-março de 2017); e duas alunas voluntárias, uma atuante no primeiro ano e outra no segundo ano do projeto. Para ser bolsista do projeto era preciso ter concluído a quarta fase do Curso de Graduação em Enfermagem.

A coleta de dados ocorreu por meio de aplicação de questionário contendo perguntas abertas e fechadas. Perguntas abertas: qual a sua percepção, como bolsista de extensão, sobre as atividades realizadas e a aprendizagem alcançada no desenvolvimento do referido projeto de extensão. Quais atividades você realizou durante o projeto? Qual a sua avaliação sobre a atividade de extensão realizada? Perguntas fechadas: idade, fase do curso que estava cursando quando da participação no projeto, experiência anterior no desenvolvimento de pesquisa, experiência anterior no desenvolvimento de projetos de extensão, experiência anterior na análise dos dados de pesquisa, experiência anterior na elaboração de manuscritos contendo resultados de projetos de pesquisa e/ou extensão.

Os bolsistas responderam ao questionário durante encontro do Grupo de Pesquisa Cuidando \& Confortando, grupo no qual frequentam. A coleta de dados ocorreu no mês de setembro de 2016. 
Atenção oncológica na atenção básica: projeto de extensão na formação de acadêmicos de enfermagem

Os dados foram submetidos à análise de conteúdo (BARDIN, 2011) e a discussão foi sustentada por estudos relacionados à temática em questão. O Termo de Consentimento Livre e Esclarecido foi aplicado e o anonimato dos participantes foi mantido pela codificação: BE 1 até BE 7, sendo a letra B de bolsista e a letra E de extensão.

\section{RESULTADOS E ANÁLISES}

As idades das bolsistas oscilaram entre 20 e 28 anos. A fase do Curso de Graduação em Enfermagem cursada durante o desenvolvimento do projeto como bolsistas oscilou entre a $5^{\mathrm{a}}$ e a $9^{a}$ fase. Em relação às experiências acadêmicas anteriores, duas bolsistas registraram participação no desenvolvimento de pesquisa, uma registrou experiência anterior no desenvolvimento de projetos de extensão, uma registrou experiência anterior na análise dos dados de pesquisa, e uma registrou experiência anterior na elaboração de manuscritos contendo resultados de projetos de pesquisa e/ou extensão.

As atividades realizadas abrangeram: elaboração de aulas para compor o OVA; busca na literatura científica para composição do banco de dados do referido projeto; apresentação do projeto aos participantes do estudo; coleta de dados juntos com os enfermeiros atuantes na atenção básica de Florianópolis para identificação das demandas de atendimento em oncologia e déficits de capacitação; processamento e análise estatística dos dados; participação na Semana de Ensino, Pesquisa e Extensão da UFSC (SEPEX) 2015; participação em oficina para aprendizagem sobre o OVA; elaboração de relatórios para a Pró-Reitoria de Extensão Universitária (Proex); participação na elaboração de dois manuscritos para divulgação dos resultados obtidos no projeto de extensão. Outra atividade desenvolvida que resultou deste projeto foi a elaboração de um Trabalho de Conclusão de Curso (TCC).

A avaliação dos bolsistas sobre as atividades realizadas e percepções sobre a aprendizagem alcançada com o desenvolvimento deste projeto foram agrupadas nas seguintes categorias temáticas:

\section{Contribuição para formação em Enfermagem}

Esta categoria temática constitui-se de algumas unidades de registros resultantes do agrupamento das ideias apontadas pelos bolsistas a contribuição do projeto para a ampliação dos conhecimentos já apreendidos, como, por exemplo, o conhecimento sobre a Oncologia e o 
Atenção oncológica na atenção básica: projeto de extensão na formação de acadêmicos de enfermagem

desenvolvimento de pesquisas; da apreensão de novos conhecimentos, sendo estes voltados ao desenvolvimento do OVA, bem como, de novas habilidades consideradas atividades inovadoras na formação dos alunos, tais como: a elaboração de aulas para compor o OVA, a coleta de dados e análise dos dados da pesquisa.

Além disto, os bolsistas citaram a possibilidade de aprimoramento do trabalho em equipe, da contribuição da atividade de pesquisa e extensão no desenvolvimento do TCC, visto que, o projeto oportunizou experiência, facilitando o desenvolvimento da pesquisa para a elaboração da atividade acadêmica obrigatória mencionada. E ainda, o projeto também expôs os alunos à divulgação dos resultados do projeto de pesquisa em eventos científicos. A SEPEX foi citada como uma oportunidade ímpar, pelo enfrentamento do público, exposição de ideias. A seguir apresenta-se alguns depoimentos dos bolsistas:

A participação neste projeto possibilitou maior aprendizado sobre o tema e sobre as tecnologias que podemos utilizar [...] (BE1).

A participação como bolsista ampliou men aprendizado sobre projetos de extensão, assim como sobre conhecimentos relacionados a Oncologia, e uma análise do déficit de conhecimentos dos enfermeiros atuantes na Atenção Básica. [...] Também deste projeto produð̨i o meu trabalbo de conclusão do curso, na qual fiz a análise dos resultados de um recorte do questionário aplicado (BE2).

$\mathrm{Na}$ minha concepção, as aulas que ajudei a montar são realmente muito importantes, [...] para melhor qualificaşão de tais profissionais (BE3).

Com a participação no projeto de extensão consegui presenciar, aprender e executar as etapas que compõem uma pesquisa, e essa vivência me ajudou, posteriormente, quando iniciei meu trabalho de conclusão de curso $[\ldots]$ (BE4).

Por ter participado desde a coleta de dados até a análise e construção do manuscrito, tive um grande aprendizado no que diz respeito à realização de uma pesquisa por completa (BE5).

Expor o projeto na semana de ensino, pesquisa e extensão assim como para os participantes da pesquisa também foi uma ótima experiência acadêmica por se tratar de um momento de exposição de ideias onde é necessária uma postura diferente como acadêmica (BE5).

Trabalhar em grupo proporcionou um crescimento pessoal e profissional, assim como a aproximação com os profissionais da atenção básica. Foi um período de grande aprendizado e envolvimento (BE5).

Para acadêmicos, vívidos em buscar conhecimentos, as oportunidades de relação da teoria com a prática são vistas como momento especial, pois nessas oportunidades eles aplicam o conhecimento apreendido, identificam e buscam novas necessidades de aprendizagem. Neste projeto, os professores foram estimuladores, mas as demandas vindas da prática estimularam ainda mais o interesse e a participação dos alunos, num processo contínuo de aplicação e busca 
Atenção oncológica na atenção básica: projeto de extensão na formação de acadêmicos de enfermagem

pelos novos saberes, incluindo a elaboração de estratégias para facilitar a educação permanente dos profissionais atuantes na atenção básica.

Durante a formação profissional, a articulação entre ensino, pesquisa e extensão, promove o desenvolvimento de competências e habilidades necessárias à construção de um novo perfil de profissional enfermeiro, capaz de atuar considerando os problemas e/ou situações de saúde, incluindo as doenças mais prevalentes no perfil epidemiológico nacional, principalmente no caso de uma doença como o câncer. (FREITAS et al., 2014).

O desenvolvimento da capacidade de produzir conhecimento próprio, assegurando uma assistência de qualidade e estruturada dentro do rigor científico, tem como principal pilar, a articulação da pesquisa com o ensino e os serviços de saúde. Sendo assim, deve haver uma integração mais ampla, que envolva também a pesquisa, reconhecendo o potencial educativo da mesma na formação e na educação permanente dos trabalhadores da saúde. (ELLERY; BOSI; LOIOLA, 2012). De acordo com a lógica da formação profissional e, considerando as demandas da sociedade, a saúde requer profissionais que aprendam a aprender, tornando suas práticas cuidadoras a partir do desenvolvimento de si, do coletivo e da instituição. Neste contexto, as ações de extensão e as transformações resultantes da educação permanente objetivam a atuação crítica, propositiva, compromissada e tecnicamente competente. (FREITAS et al., 2014).

Nessa perspectiva, preconiza-se que o processo educativo proporcionado pela pesquisa e extensão, não deve ser considerado um momento particular da vida acadêmica, e sim, um investimento na formação para o trabalho, no qual o mesmo possa definir as demandas educacionais. (FREITAS et al., 2014).

\section{Contribuição para a educação permanente em enfermagem e para a atenção oncológica na atenção básica em saúde}

Esta categoria temática constitui-se da percepção dos alunos quanto aos benefícios do projeto de extensão, que incluem: o aprimoramento técnico e científico dos profissionais associados à diminuição dos déficits de qualificação dos profissionais para o cuidado do paciente com câncer, ou com suspeita de câncer, por meio da educação permanente a distância com o uso da tecnologia da informação e da comunicação, neste caso, com a criação e disponibilização do OVA. Os alunos ainda apontaram que o aprimoramento dos profissionais e a praticidade do uso do OVA como estratégia de formação teve como maior beneficiário o usuário do SUS, pela melhoria no cuidado recebido pelos profissionais. Alguns depoimentos selecionados para representar esta categoria: 
Atenção oncológica na atenção básica: projeto de extensão na formação de acadêmicos de enfermagem

São de extrema importância as atividades de extensão e pesquisa realizadas neste projeto, visto que tem por objetivo aprimorar o atendimento do enfermeiro ao paciente oncológico [...] (BE6).

Reconheço a importância de a Universidade realizar tais ações, para melhorar a assistência prestada aos usuários do SUS. O conhecimento produzido por discentes e docentes deve ser passado adiante, pois se não houver nada vale a pesquisa. Acredito que a atividade realizada foi muito importante, pois simplificou o acesso de enfermeiros aos conbecimentos sobre diversos temas dentro da oncologia [...] também reforçou a importância da parceria entre a Universidade e os demais campos de trabalho do enfermeiro (BE1).

Acredito que o projeto ajudará muito, pois as informações necessárias para sanar suas dúvidas estarão disponiveis em um clique de forma fácil e entendivel, além de outras bibliografias, manuais e portarias que abrangem a oncologia e o paciente com câncer (BE4).

A educação permanente, presencial e/ou a distância, é discutida largamente por sua importância e necessidade na transformação dos serviços de saúde e da saúde dos usuários do SUS. Porém, a elevada demanda de trabalho existente, dentre outros fatores, dificulta sua realização efetiva.

O uso do ambiente virtual de aprendizagem foi visto pelos alunos como uma ferramenta que favorece a educação permanente, mas, principalmente a aprendizagem para a atenção oncológica. Os relatos mostraram claramente que para este cuidado há déficit de qualificação e, considerando a epidemiologia do câncer, a atenção básica como porta de entrada para os atendimentos de saúde, e ainda, considerando os danos ocasionados pelo câncer sobre o indivíduo e sociedade, é urgente que esta realidade seja modificada.

A educação permanente possibilita preservar a qualificação do serviço de forma continuada, visto que amplia as relações entre ensino e serviço e entre a docência e a atenção à saúde. É caracterizada pelo processo de aprendizagem no ambiente de trabalho, nos significados e na possibilidade de transformar a prática. (FREITAS et al., 2014).

Paralelamente à necessidade de educação permanente nos serviços de saúde, o Ministério da Saúde institui, em 2004, a Política Nacional de Educação Permanente em Saúde (PNEPS), objetivando a articulação do sistema de saúde com instituições formadoras, a fim de desenvolver processos educativos que respondam à realidade e às necessidades do SUS. (THULER; BERGMANN; FERREIRA, 2011).

Neste cenário, as políticas de educação permanente para os profissionais da saúde pública no Brasil que atuam na atenção oncológica merecem especial importância. A utilização dos recursos da educação à distância, especialmente com o uso de ambientes virtuais de aprendizagem, pode vir a representar uma promissora estratégia de superação dos desafios que se apresentam no contexto destas políticas. (SILVA, 2015). 
Atenção oncológica na atenção básica: projeto de extensão na formação de acadêmicos de enfermagem

Ressalta-se que, em 2005 foi publicada pelo Ministério da Saúde a Política Nacional de Atenção Oncológica (PNAO) (BRASIL, 2005), sendo que esta política destacava a qualificação, a especialização e a educação permanente como componente essencial para a estratégia do controle do câncer em nível nacional. (THULER; BERGMANN; FERREIRA, 2011). Esta proposição se manteve confirmada na Portaria n. 874/2013, que substituiu a PNAO. (BRASIL, 2013).

Para auxiliar no processo educativo de ampliação da qualificação das ações de saúde, Costa et al.. (2011) afirma que o uso da tecnologia como o ambiente virtual de aprendizagem, oferece novos caminhos para o processo de aprendizado através da integração de diversas linguagens e recursos, bem como para a mediação de saberes, sendo determinante para a formação acadêmica e, consequentemente, para o aumento da qualidade do mercado de trabalho.

Evidencia-se com esta perspectiva desafiadora a área de educação em enfermagem, em razão do avanço das tecnologias da informação e comunicação (TIC) nos espaços acadêmicos e da necessidade de ampliação no atendimento das demandas de qualificação em saúde. Os recursos disponibilizados pelas TICs, em especial os ambientes virtuais de aprendizagem favorecem o processo de construção do conhecimento e interação entre alunos, professores, tutores e profissionais, enriquecendo o compartilhamento de ideias e possibilitando uma aprendizagem dinâmica e colaborativa. (COSTA et al., 2011).

\section{Problematizando a atuação do enfermeiro na atenção básica em saúde e a formação acadêmica}

Esta categoria agrupa os achados que apontam o déficit da formação em Oncologia e o quanto isto é prejudicial para o atendimento do usuário do SUS. Mas, apesar deste déficit, a recente formação apresenta-se com melhor qualidade, quando comparado com os déficits de qualificação apontados pelos enfermeiros atuantes na atenção básica em saúde, na percepção dos bolsistas. Ainda como problematização, foi apontado que o ritmo de trabalho vem exigindo acesso objetivo e rápido às informações e, o projeto de extensão possibilitou isto aos enfermeiros e outros profissionais da enfermagem na atenção básica em saúde do município de FlorianópolisSC.

A seguir apresenta-se alguns depoimentos para representar a categoria:

Foi um processo positivo, pois como acadêmica pude perceber o quanto nosso ensino é bom, pois me permitiu conhecer melhor sobre a doença câncer, seus tratamentos e afins e, me permitiu responder de forma tranquila os questionários, enquanto os profissionais muitas vezes não tinham uma noção do que estava sendo questionado (BE7). 
Atenção oncológica na atenção básica: projeto de extensão na formação de acadêmicos de enfermagem

Durante a graduação temos apenas algumas aulas relacionadas à oncologia, na quarta fase. [...] $O$ conteúdo sobre oncologia dado na graduação é pouquíssimo (BE1).

A atividade de extensão demonstrou a existência das necessidades dos enfermeiros diante da atenção oncológica, reforçando a importância de bases confiáveis e de fácil acesso para que eles possam acessar e obter as informacõoes necessárias de forma rápida e com conteúdo direcionado à Enfermagem (BE2).

De acordo com os resultados, o conbecimento dos enfermeiros que atuam na atenção básica é escasso sobre Oncologia, tratamentos e seus efeitos colaterais (BE4).

O discutido na última categoria já aponta a necessidade de qualificação. A educação permanente sempre será necessária, entretanto, se os currículos de formação incluírem conteúdos teóricos e práticos mínimos sobre Oncologia, o déficit de qualificação existente poderá ser minimizado, consequentemente, o usuário do SUS será cuidado com melhor e maior atenção e saber científico.

Apesar das crescentes ações de educação em saúde empreendidas pelo Ministério da Saúde e pelo INCA, ainda há considerada distância dessas ações entre a função institucional e a oferta de soluções para a educação permanente dos profissionais que atuam na atenção oncológica. Não cabe ao Ministério da Saúde apenas ofertar capacitações e treinamentos para atender as necessidades de qualificação, mas também de promover consultoria e soluções adequadas de capacitação e formação para sanar os déficits existentes na atenção oncológica. (SILVA, 2015).

E ainda, cabem as instituições de ensino a qualidade da formação, esta voltada às principais demandas sociais do país, numa articulação coesa com os governos em prol do desenvolvimento das profissões e da população.

Como limite deste estudo registra-se que a elaboração dos resultados aqui apontados não inclui as atividades finais do projeto de extensão, tais como: avaliação do OVA pelos coordenadores dos Distritos Sanitário de Saúde e profissionais de enfermagem.

\section{CONSIDERAÇÕES FINAIS}

Este estudo mostrou a percepção dos acadêmicos de enfermagem diante da participação como bolsistas de um projeto de extensão. Esse projeto abrangeu as demandas de educação permanente para atenção oncológica na atenção básica de Florianópolis.

Os bolsistas apontaram a efetividade da participação em projeto de extensão como estratégia formativa, além disto, apontaram os benefícios do projeto de extensão para as 
Atenção oncológica na atenção básica: projeto de extensão na formação de acadêmicos de enfermagem

instituições de saúde, destacando o uso dos ambientes virtuais de aprendizagem para efetivação da educação permanente em saúde e qualificação dos profissionais.

Recomenda-se a divulgação dos resultados alcançados pelos projetos de extensão desenvolvidos pelas instituições de ensino, como o que pretende-se com este artigo.

\section{AGRADECIMENTOS}

Este estudo somente foi possível ser desenvolvido pela ação conjunta de professoras do Departamento de Enfermagem da UFSC e acadêmicas do Curso de Graduação em Enfermagem no projeto de extensão aqui retratado. Assim, além das autoras citadas neste artigo registra-se a colaboração da Professora Francis Tourinho e das acadêmicas Rafaela Dutra Nunes da Silva, Gabriela Schutz da Silva, Bárbara Franz Patrício, Mayara C. Fontão e Anizelle Aline Lopes.

\section{REFERÊNCIAS}

BARDIN, Laurence. Análise de conteúdo. São Paulo: Edições 70, 2011.

BRASIL. Ministério da Saúde. Portaria n. 874, de 16 de maio de 2013. Institui a Política Nacional para a Prevenção e Controle do Câncer na Rede de Atenção à Saúde das Pessoas com Doenças Crônicas no âmbito do Sistema Único de Saúde (SUS). Diário Oficial da União. 17 mai. 2005.

BRASIL. Ministério da Saúde. Portaria n. 2.439/GM, de 8 de dezembro de 2005. Institui a Política Nacional de Atenção Oncológica: Promoção, Prevenção, Diagnóstico, Tratamento, Reabilitação e Cuidados Paliativos, a ser implantada em todas as unidades federadas, respeitadas as competências das três esferas de gestão. Diário Oficial da União. 9 dez. 2005.

COSTA, Priscila Berenice et al. Fluência digital e uso de ambientes virtuais: caracterização de alunos de enfermagem. Revista Escola de Enfermagem Usp, São Paulo, v. 45, n. 1, p.94-1589, nov. 2011. Disponível em: <http://www.scielo.br/scielo.php?script=sci_arttext\&pid=S008062342011000700008>. Acesso em: 15 out. 2016.

ELLERY, Ana Ecilda Lima; BOSI, Maria Lúcia Magalhães; LOIOLA, Francisco Antonio. Integração Ensino, Pesquisa e Serviços em Saúde: antecedentes, estratégias e iniciativas. Revista Saúde e Sociedade, São Paulo, v. 22, n. 1, p.187-198, out. 2012. Disponível em: $<$ http://www.scielo.br/scielo.php?script=sci_arttext\&pid=S0104-12902013000100017> Acesso em: 15 out. 2016.

ERVIK, M. et al. Cancer Today. Lyon, France: International Agency for Researchon Cancer. Cancer Today, 2016. Disponível em: <http://gco.iarc.fr/today>. Acesso em: 1 set. 2016. 
Atenção oncológica na atenção básica: projeto de extensão na formação de acadêmicos de enfermagem

FREITAS, Tiago Luan Labres de et al. Extensão Universitária: contribuições para a formação profissional do enfermeiro. Revista Eletrônica de Extensão da Uri, Rio Grande do Sul, v. 10, n. 18, p.164-173, mai. 2014.

INSTITUTO NACIONAL DE CÂNCER JOSÉ ALENCAR GOMES DA SILVA. Coordenação de Prevenção e Vigilância Estimativa 2016: incidência de câncer no Brasil. Rio de Janeiro: INCA, 2015.

LUZ, K. R. et al. Estratégias de enfrentamento por enfermeiros da oncologia na alta complexidade. Rev. Bras Enferm [Internet]. 2016; 69(1):59-63. Disponível em: $<$ http:/ /www.scielo.br/scielo.php?script=sci_arttext\&pid=S0034-

$71672016000100067 \& \operatorname{lng}=$ pt\&tlng=pt $>$. Acesso em: 17 ago. 2017.

LUZ, K. R. et al. Problemas éticos vivenciados por enfermeiros oncológicos. Rev. Latino-Am. Enfermagem nov.-dez. 2015; 23(6): 1187-94. Disponível em:http://www.scielo.br/pdf/rlae/v23n6/pt_0104-1169-rlae-23-06-01187.pdf. Acesso em: 17 ago. 2017

PRANKE, Amanda; FRISON, Lourdes Maria Bragagnolo. Potencialização da Aprendizagem Autorregulada de Bolsistas do PIBID/UFPel do curso de Licenciatura em Matemática através de Oficinas Pedagógicas. Bolema: Boletim de Educação Matemática,[s.l.], v. 29, n. 51, p.223240, abr. 2015. FapUNIFESP (SciELO). Disponível em: $<$ http://www.scielo.br/scielo.php?script=sci_arttext\&pid=S0103-

636X2015000100013\&lng=pt\&nrm=iso\&tlng=en >. Acesso em: 14 nov. 2016.

SILVA, Marcelo Camacho. Atenção Oncológica do Colo de Útero no Brasil: as Políticas de Educação à Distância na Educação Permanente Profissional. Revista de Ensino, Educação e Ciências Humanas, Londrina, v. 16, n. 3, p.234-244, 2015. Disponível em: < http://www.pgsskroton.com.br/seer/index.php/ensino/article/view/3110>. Acesso em: 15 out. 2016.

SOUZA, Geize Rocha Macedo de; CAZOLA, Luiza Helena de Oliveira; OLIVEIRA, Sandra Maria do Valle Leone de. Atuação dos enfermeiros da estratégia saúde da família na atenção oncológica. Esc. Anna Nery, Rio de Janeiro , v. 21, n. 4, e20160380, 2017 . Disponível em: http:/ /www.scielo.br/scielo.php?script=sci_arttext\&pid=S1414-

81452017000400207\&lng=en\&nrm=iso. Acesso em: 17 ago. 2017.

THULER, Luiz Claudio Santos; BERGMANN Anke; FERREIRA, Solange Canavarro. Ensino em Atenção Oncológica no Brasil: Carências e Oportunidades. Revista Brasileira de Cancerologia, Rio de Janeiro,v.57, n.4, p. 467-472, 2011. 